\title{
人类可溶性环氧化物水解酶抑制剂的研究进展
}

\author{
黄少胥 王 勇 龙亚秋* \\ (中国科学院上海药物研究所合成化学实验室 上海 201203)
}

\begin{abstract}
摘要 高血压是现代人的常见疾病. 虽然有很多不同机理的降压药在临床使用, 但是由于个体的差异性, 高血压的治 疗越来越倾向于个体化治疗，因此降压药的使用也不仅仅局限于血压的降低. 人类可溶性环氧化物水解酶抑制剂最大 的优势在于其在降压的同时还具有显著的抗炎作用. 详细地阐述了人类可溶性环氧化物水解酶抑制剂从早期的环氧结 构类型到第三代脉类结构的发展过程和近期研究进展.
\end{abstract}

关键词 可溶性环氧化物水解酶; 花生四烯酸环氧化物; 抑制剂; 1,3-二取代衤; 高血压; 炎症

\section{Advances in the Research of Human Soluble Epoxide Hydrolase Inhibitors}

\author{
Huang, Shaoxu Wang, Yong Long, Yaqiu* \\ (Laboratory of Medicinal Chemistry, Shanghai Institute of Materia Medica, Shanghai Institutes for Biological Sciences, \\ Chinese Academy of Sciences, Shanghai 201203)
}

\begin{abstract}
Hypertension is a common illness nowadays. Although a wide variety of antihypertensive agents are available in the market for the treatment of hypertension, the antihypertensive treatment tends to be individualized, and the treatment should exceed the limitation of lowering blood pressure only. Compared to other antihypertensive agents, the advantage of human soluble epoxide hydrolase inhibitors (HsEHIs) is that they can not only reduce the blood pressure, but also have remarkable anti-inflammatory effects. Herein, the development of human soluble epoxide hydrolase inhibitors is reviewed from early epoxide HsEHIs to the third generation of urea HsEHIs.

Keywords soluble epoxide hydrolase; epoxyeicosatrienoic acid; inhibitors; 1,3-disubstituted urea; hypertension; inflammation
\end{abstract}

环氧化合物(或称作氧杂环丙烷)在自然界及人体中 广泛存在. 在体内, 许多芳香和烯类化合物通常代谢成 环氧化合物 ${ }^{[1 \sim 3]}$. 环氧化合物高度极化的氧一碳键以及 高度张力的环系使其具有特殊的反应形式, 一些活泼环 氧化合物易于与关键的生物靶点(例如 DNA 和蛋白质) 发生亲电反应、导致突变等. 因此, 环氧化合物在体内 的含量要保持在特定的水平 ${ }^{[4]}$, 这是通过适当的转换进 行调节的. 环氧化物水解酶(epoxide hydrolase)催化环氧 化合物水解成相应的 1,2-二醇就是细胞转化环氧化合物 的主要途径之一 ${ }^{[5]}$.

环氧化物水解酶在体内主要发挥三个作用: 解毒、 代谢和信号分子调节. 在哺乳动物中存在许多种环氧化 物水解酶, 包括胆固醇环氧化物水解酶 $(\mathrm{chEH})^{[6] \text { 、羟基 }}$
环氧素水解酶 ${ }^{[7]}$ 、微粒体环氧化物水解酶 $(\mathrm{mEH})$ 以及可 溶性环氧化物水解酶(sEH). 最近的研究表明, sEH 在调 节血压和治疗炎症中发挥重要的作用，抑制 sEH 可能成 为治疗高血压、动脉硬化症、中风及肾衰竭等疾病的有 效方法 ${ }^{[8]}$.

可溶性环氧化物水解酶 $\mathrm{sEH}$ 直接参与花生四烯酸 环氧化物(EETs)和亚油酸环氧化物的代谢 ${ }^{[11,12]}$. EETs 是 一类内源性的化学介质，它作用于血管、肾及心脏，调 节血压. EETs 的血管扩张性能与钾离子通道开放态几 率的增加有关，该通道由镉离子激活，并且最终导致血. 管平滑肌的超极化. 实验证明, 一旦花生四烯酸环氧化 物被水解成相应的邻二羟基化合物，其调节血压的能力 丧失 ${ }^{[13]}$. 而且, 原发性高血压鼠或血管紧张素 II 诱导的

\footnotetext{
*E-mail: yqlong@mail.shcnc.ac.cn

Received July 13, 2011; revised November 17, 2011; accepted December 7, 2011.

Project supported by the National Natural Science Foundation of China (Nos. 81021062, 81072527).

国家自然科学基金(Nos. 81021062,81072527)资助项目。
} 
高血压鼠服用 $\mathrm{sEH}$ 抑制剂, 都观察到鼠血压的降低 ${ }^{[8,14]}$. 另外, 比较野生型小鼠和敲除 $\mathrm{sEH}$ 基因小鼠的血压, 也 发现前者要高于后者 ${ }^{[15]}$. 这些现象进一步证明了 $\mathrm{sEH}$ 在 调节血压中的作用, 说明可溶性环氧化物水解酶抑制剂 是治疗高血压的一种潜在的新方法 ${ }^{[16]}$.

目前成功应用于临床治疗的降压药主要有利尿剂、 $\beta$-受体阻滞剂、血管紧张素转换酶抑制剂、血管紧张素 受体阻滞剂、钙离子通道阻滞剂、 $\alpha$-受体阻滞剂以及肾 素抑制剂等, 但由于大部分的高血压患者常常同时伴随 其它疾病, 例如糖尿病、冠状动脉疾病、高血脂以及代 谢综合征等，所以，许多临床实验也在探索不同机制的 降压药是否产生不同的临床结果. 一项研究结果发现, 相较于钙离子通道阻滞剂氨氯地平(amlodipine)和血管 紧张素转换酶抑制剂赖诺普利(lisinopril)等降压药, 噻 嗪类利尿药氯噻酮(chlorthalidone)在预防心血管疾病方 面更加优异，但应用 $\beta$-受体阻滞剂多沙唑嗪(doxazosin) 却有可能增加患心血管疾病的风险 ${ }^{[17]}$. 因此, 对于高血. 压患者应该给予个体化治疗，而且也不应局限于血压的 降低. 另外，一部分的高血压患者需要多种药物共同作 用以达到合适的血压值, 这就需要更多不同作用机理的 药物, 以便得到更加合适并且风险较低的药物组合.

鼠模型实验显示, sEH 抑制剂( $\mathrm{sEHI}$ )不仅在降低炎 症廿烷类(eicosanoid)物质(例如前列腺素 $\mathrm{E}_{2}$ )较非甾体抗 炎药(nonsteroidal anti-inflammatory drugs, NSAIDs)更加 有效, 甚至还能增强 NSAIDs 的抗炎作用 ${ }^{[18]}$. 一项可溶 性环氧化物水解酶抑制剂与吲哚美辛(indomethacin)、塞 来昔布(celecoxib)以及 Rofecoxib 等非甾体抗炎药联合 使用的实验显示, $\mathrm{sEHI}$ 不仅能够降低 NSAID 的用量, 而 且还能有效地降低血压 ${ }^{[18]}$. 因此, 对那些需要高剂量服 用非甾体抗炎药的患者联合施以可溶性环氧化物水解 酶抑制剂将极具吸引力. 总之, 可溶性环氧化物水解酶 抑制剂相较于其它降压药最显著的优势就在于其在降 压的同时还具有显著的抗炎作用.

\section{1 人类可溶性环氧化物水解酶(HsEH)抑制剂 研究进展}

\section{1 早期的 HsEH 抑制剂}

最早被发现、设计的 HsEH 抑制剂是一些含有环氧 结构的化合物 (图 1) ${ }^{[19,20]}$. 它们中的大多数实际上是作 为环氧化物水解酶的底物, 只不过被水解的速度相对较 慢 ${ }^{[21]}$. 然而, 这些化合物内在的不稳定性(尤其是当存 在谷胱甘肽时)使得它们在体外只有短暂的抑制效果, 而且在细胞及体内无效 ${ }^{[19]}$. 值得注意的是, sEH 也可以 被 $\mathrm{Zn}^{2+}$ 抑制 ${ }^{[22]}$, 这可能是由于其 $\mathrm{N}$-端连接 $\mathrm{Mg}^{2+}$ 的区域 被 $\mathrm{Zn}^{2+}$ 取代, 从而导致了活性的丧失(在炎症发生过程
中, 肝中二价金属离子的浓度, 尤其是 $\mathrm{Zn}^{2+}$ 的浓度通常 都会升高 ${ }^{[23]}$, 这表明, 结合 $\mathrm{Zn}^{2+}$ 可能是人体降低 $\mathrm{HsEH}$ 活性的自主途径 ${ }^{[86,12]}$.

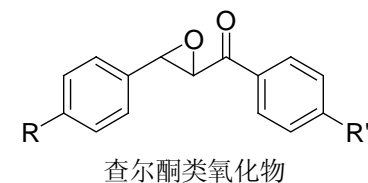

图 1 早期发现的 HsEH 抑制剂结构示例
需要指出的是, 测定 $\mathrm{HsEH}$ 抑制剂活性 $\left(\mathrm{IC}_{50}\right.$ 值)的 方法主要有两种. 最早是应用消旋的反式-2,3-环氧-3苯基丙基碳酸-4-硝基苯基酯(4-nitrophenyl-trans-2,3epoxy-3-phenylpropyl carbonate, NEPC)作为分光光度底 物的测定方法 ${ }^{[24]}$. 后来发展了应用反-[(3-苯基环氧乙 烷-2-基)甲基]-[氧基(6-甲氧基萘-2-基)]甲酯(CMNPC) 为底物基于荧光的测试方法 ${ }^{[25]}$. 前一种基于 NEPC 的方 法的灵敏度较低，一般仅能达到微摩尔 $\left(\mu \mathrm{mol} \cdot \mathrm{L}^{-1}\right)$ 水平; 而应用 CMNPC 为底物基于荧光的测试方法, 灵敏度较 高, 通常能够精确到纳摩尔水平 $\left(\mathrm{nmol} \cdot \mathrm{L}^{-1}\right)$, 可以准确 区分高活性化合物.

\section{2 第一代 HsEH 抑制剂}

针对 EHs 的机理研究表明, 酶的催化位点存在一个 关键的天冬氨酸残基 ${ }^{[4,21]}$, 因此, 最早的 HsEH 抑制剂研 究主要集中在羧基修饰试剂上.

二环己基碳化二亚胺(DCC) 是酰胺键以及肽合成中 常用的试剂，它能很容易地与羧酸反应，生成相应的活 化酯. 以 DCC 作为人环氧化物水解酶抑制剂取得了很 好的结果. 然而, 随后的药动学研究发现, 在使用的缓 冲溶液中, DCC 迅速水解成相应的嫝: $N, N$-二环已基腿 (DCU) (Eq. 1)，因此，水解产物 DCU 才是真正起到抑制 $\mathrm{HsEH}$ 作用的化合物 ${ }^{[26]}$. 环氧化合物的氧带有部分负电 荷, 碳上带有部分正电荷, 这和嫝类化合物结构的电荷 空间分布类似(Eq. 1).

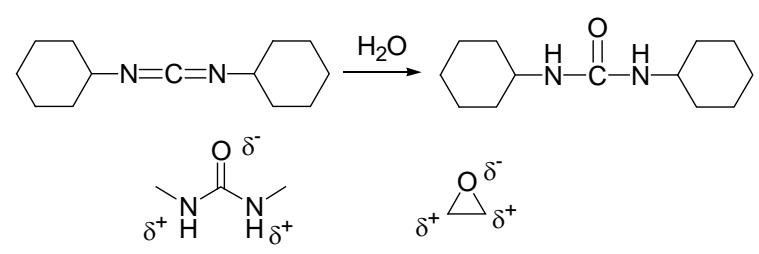

为了优化抑制剂的药效团，一些腿结构的类似物被 合成出来, 主要是将腿官能团中的氮和氧原子用其它的 原子替换(表 1$)^{[12]}$. 当其中的一个氮原子分别置换成氧、 碳或硫原子时，只有氧原子取代衍生物 4 活性略微提高 (对照化合物二环已基艮 3 与化合物 4 6). 但是, 如果 
将脲中的两个氮原子全部替换成碳或氧原子, 活性急剧 下降(化合物 7 9). 另外, 脲官能团中的羰基氧也对活 性有重要作用, 脲中的氧置换成硫原子(化合物 10)或者 亚胺(化合物 11)，都导致了活性的丧失.

表 1 一些药效团对 HsEH 的抑制活性

Table 1 Inhibition of HsEH by several pharmacophores

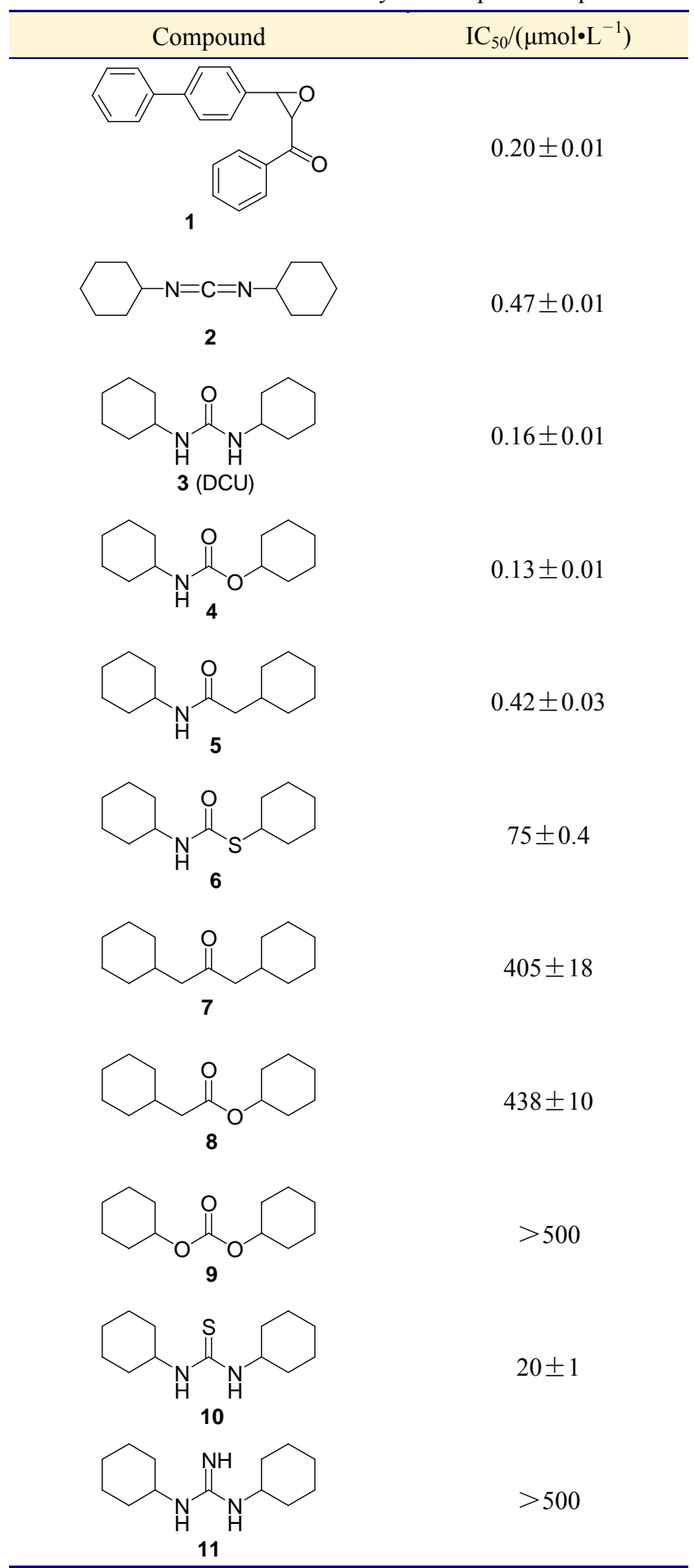

Hammock 课题组 ${ }^{[12]}$ 对嫝官能团中氮原子上取代基 对活性的影响进行了细致的考察(表 2). 观察化合物
12 17, 从 1,3-无取代到四取代脲, 只有 1,3-二取代的 化合物 15 和 1,1,3-三取代的化合物 16 有活性. 这表明 要保留活性, 至少需要嫝中的一个氮原子上存有氢原 子. 而在嫝中至少一个氮原子上存有氢原子的化合物中 $(15,16,18 \sim 21)$, 脲两侧为大的取代基时, 活性相对较 好，其中 $N$-环已基- $N^{\prime}$-金刚烷-1-基脲(21)在这一系列中 具有最好的抑制 HsEH 的体外活性.

表 2 脲结构中 $\mathrm{N}$ 原子上的取代基对活性的影响 ${ }^{a}$

Table 2 Exploring the effect of $N$-substitution of urea derivatives

(1)

${ }^{a}$ 活性测试采用了基于 NEPC 的测定方法.

试验表明脲类的环氧化物水解酶抑制剂在抑制过 
程中没有被 $\mathrm{HsEH}$ 转化, 计算数据和晶体结构表明它们 与环氧化合物和酶的作用位点相同. 在羧酸酯酶、谷胱 甘肽转移酶、环氧水解酶、四种 $\mathrm{P} 450$ 以及辅酶 $\mathrm{A}$ 氧化 酶中, 二环已基嫝(3)只对 HsEH 起抑制剂作用, 表明这 类脲类化合物对可溶性环氧化物水解酶具有较好的选 择性.

但是，这些二烷基脲具有高的晶格能、高的熔点， 使得这类化合物的水溶性极差, 甚至在有机溶剂中的溶 解性也不理想. 这些物理性质有可能影响这类环氧水解 酶抑制剂在体内的效果, 也给药物的制剂带来困难. 例 如 $N$-环已基- $N^{\prime}$-金刚烷-1-基嫝(21)的体外 $\mathrm{IC}_{50}$ 值为 0.12 $\mu \mathrm{mol} \cdot \mathrm{L}^{-1}$ ，但在体内却没有活性. 为了改善这类䐂类 $\mathrm{HsEH}$ 抑制剂的成药物理性质, Hammock 小组 ${ }^{[27,28]}$ 以化 合物 21 为先导化合物, 对其进行一系列的结构改造. 一 方面保留化合物 21 中的一个环己基, 对脲上另一氮原 子上的烷基取代基进行替代, 包括从三元环到十二元环 的取代基篮选 ${ }^{[27]}$. 结果显示, 用小环替换会导致活性的 降低, 中环及大环(例如十二元环)则使活性保持. 在这 一系列的替换中, 金刚烷基的取代物是活性最好的化合 物(化合物 21). 这说明脲类 $\mathrm{sEH}$ 抑制剂的一端需要大体 积的疏水性基团有利于活性的发挥.

\section{3 第二代 HsEH 抑制剂}

由于疏水性的增加有利于活性的提高, 而脂肪链的 疏水性要强于苯环, 因此 Hammock 小组在保留化合物 21 中的一个环已基的基础上, 将腿的另一取代基置换 成柔性的长链, 并在长链末端加入极性基团以期提高水 溶性. Hammock 小组 ${ }^{[27,28]}$ 尝试了从乙酸及乙酸甲酯到正 十二酸的取代(表 3), 结果发现, 当柔性的碳链长度小于 6 时，自由的羧酸基团对活性不利(化合物 22, 24, 26 和 28); 随着碳链的增长 $(>6)$, 碳链末端的自由羧酸基团 对活性的消极影响减弱. 这就为在保持抑制剂活性的基 础上提高化合物的水溶性提供了一条可行的途径, 最后 得到的金刚烷-1-基的腿 38 的水溶性和活性都是最高的.

从表 3 可以看到，当一侧的取代基为丁酸甲酯时 (化合物 27), 活性大幅度提高 $\left(\mathrm{IC}_{50}=6.2 \pm 0.5 \mu \mathrm{mol} \cdot \mathrm{L}^{-1}\right)$, 这说明在距嫝基团羰基第五原子处(约 $7.5 \AA$ )引入极性 官能团将有利于活性的提高，而极性基团的引入则有可 能改善化合物的水溶性. 一般来说, 在亲脂性抑制剂中 加入极性基团, 常常导致活性的急剧下降, 因为围绕在 极性官能团周围的水壳(water shell)减弱了抑制剂与活 性位置的结合. 但是，倘若新加入的极性基团能够与酶 在活性位点形成氢键并且不会干扰主要药效团与酶的 结合, 那么形成氢键的能量足以补偿除去水壳所需要的 能量 ${ }^{[28]}$. 因此, 以合适的方式拼加一个能与酶相互作用 的额外的极性基团，通常能够在不影响抑制剂活性的同
表 3 短脂肪链末端置入极性酸及其相应甲酯结构的脲类衍 生物对活性的影响 ${ }^{a}$

Table 3 Exploring the effect of the addition of polar acid and corresponding methyl ester moieties onto short aliphatic chains of urea derivatives
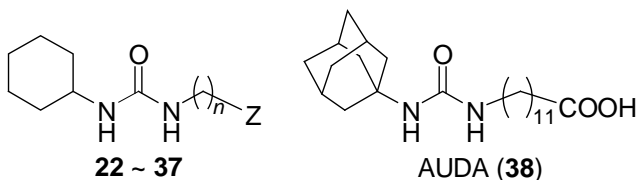

\begin{tabular}{cclc}
\hline Compound & $n$ & \multicolumn{1}{c}{$\mathrm{Z}$} & $\mathrm{IC}_{50}{ }^{b} /\left(\mu \mathrm{mol} \cdot \mathrm{L}^{-1}\right)$ \\
\hline $\mathbf{2 2}$ & 1 & $\mathrm{COOH}$ & $>500$ \\
$\mathbf{2 3}$ & 1 & $\mathrm{COOCH}_{3}$ & $70 \pm 6$ \\
$\mathbf{2 4}$ & 2 & $\mathrm{COOH}$ & $358 \pm 2$ \\
$\mathbf{2 5}$ & 2 & $\mathrm{COOCH}_{3}$ & $78 \pm 4$ \\
$\mathbf{2 6}$ & 3 & $\mathrm{COOH}$ & $>500$ \\
$\mathbf{2 7}$ & 3 & $\mathrm{COOCH}$ & $6.2 \pm 0.5$ \\
$\mathbf{2 8}$ & 5 & $\mathrm{COOH}$ & $253 \pm 8$ \\
$\mathbf{2 9}$ & 5 & $\mathrm{CH}_{3}$ & $221 \pm 2$ \\
$\mathbf{3 0}$ & 5 & $\mathrm{COOH}$ & $91000 \pm 1000$ \\
$\mathbf{3 1}$ & 6 & $\mathrm{COOH}$ & $14700 \pm 200$ \\
$\mathbf{3 2}$ & 7 & $\mathrm{COOH}$ & $1100 \pm 100$ \\
$\mathbf{3 3}$ & 7 & $\mathrm{COOCH}_{3}$ & $361 \pm 9$ \\
$\mathbf{3 4}$ & 10 & $\mathrm{COOH}$ & $230 \pm 10$ \\
$\mathbf{3 5}$ & 10 & $\mathrm{COOCH}$ & $61 \pm 1$ \\
$\mathbf{3 6}$ & 11 & $\mathrm{CH}_{3}$ & $85.2 \pm 0.5$ \\
$\mathbf{3 7}$ & 11 & $\mathrm{COOH}_{3}$ & $112 \pm 1$ \\
$\mathbf{3 8}$ & & & $(69 \pm 2) \times 10^{-3}$ \\
\hline
\end{tabular}

${ }^{a}$ 化合物 22 28 的活性是应用基于 NEPC 的测试方法获得, 其余化合物的 活性则是应用基于 $\mathrm{t}$-DPPO 的方法获得. ${ }^{b}$ 针对人可溶性环氧水解酶的活 性.

时，显著增强抑制剂的特异性，也有可能改善化合物的 成药物理性质. 在这个基础上，一系列在距离主要脲药 效团 5 个碳原子处(约 $7.5 \AA$ )引入羰基的化合物被设计合 成出来，以期改善亲脂性 HsEH 抑制剂的水溶性.

在化合物 27 的基础上，Hammock 等 ${ }^{[28]}$ 还尝试了将 其艮官能团的一侧以间氯苯基替代环己基(有利于化合 物的检测和纯化); 同时保留距离主要脲药效团五个碳 原子处的羰基，考察连接羰基两侧的原子对活性的影响 (表 4). 从表 4 可以发现, $X$ 为亚甲基, $Y$ 为亚甲基或者氧 原子时(化合物 39, 40), 活性最好. 这表明在距离脲药效 团 5 个碳原子处为酯或者酮官能团时对活性最为有利, 而诸如酰胺、碳酸酯取代则活性下降. 在此基础上，保 留艮官能团一侧的取代基为环已基(化合物 48)或金刚 烷-1-基(化合物 49)，活性进一步提高(表 5)，再次证实大 的脂肪环基团是 1,3-二取代嫝的 1-位优势取代基. 同时, 这一类在距离脲基团羰基第 5 原子处(约 $7.5 \AA$ )引入极性 官能团的化合物(化合物 48 和 49)比相应的未官能团化 的脂溶性化合物, (化合物 $\mathbf{5 0}$ 和 51) 活性略微下降, 但水 溶性大大增加. 
表 4 1-(3-氯苯基)-3-(2-取代乙基)艮类化合物对 HsEH 的抑制 活性

Table 4 Inhibition of HsEH by 1-(3-chlorophenyl)-3-(2-substituted ethyl)ureas<smiles>[Z8][13CH2]NC(=O)Nc1cccc(Cl)c1</smiles>

\begin{tabular}{cccc}
\hline Compd. & $\mathrm{X}$ & $\mathrm{Y}$ & $\mathrm{IC}_{50} /\left(\mu \mathrm{mol} \cdot \mathrm{L}^{-1}\right)$ \\
\hline $\mathbf{3 9}$ & $\mathrm{CH}_{2}$ & $\mathrm{CH}_{2}$ & $2.1 \pm 0.2$ \\
$\mathbf{4 0}$ & $\mathrm{CH}_{2}$ & $\mathrm{O}$ & $2.1 \pm 0.07$ \\
$\mathbf{4 1}$ & $\mathrm{CH}_{2}$ & $\mathrm{NH}$ & $32 \pm 0.8$ \\
$\mathbf{4 2}$ & $\mathrm{O}$ & $\mathrm{CH}_{2}$ & $26 \pm 1$ \\
$\mathbf{4 3}$ & $\mathrm{O}$ & $\mathrm{O}$ & $22 \pm 1$ \\
$\mathbf{4 4}$ & $\mathrm{O}$ & $\mathrm{NH}$ & $18 \pm 0.9$ \\
$\mathbf{4 5}$ & $\mathrm{NH}$ & $\mathrm{CH}_{2}$ & $>100$ \\
$\mathbf{4 6}$ & $\mathrm{NH}$ & $\mathrm{O}$ & $>100$ \\
$\mathbf{4 7}$ & $\mathrm{NH}$ & $\mathrm{NH}$ & $>100$ \\
\hline
\end{tabular}

表 5 1-环己基/金刚烷基-3-烷基脲类化合物对 $\mathrm{HsEH}$ 的抑制 活性 $^{a}$

Table 5 Inhibition of human sEH by 1-cylclohexyl or adamantyl-3-alkylureas

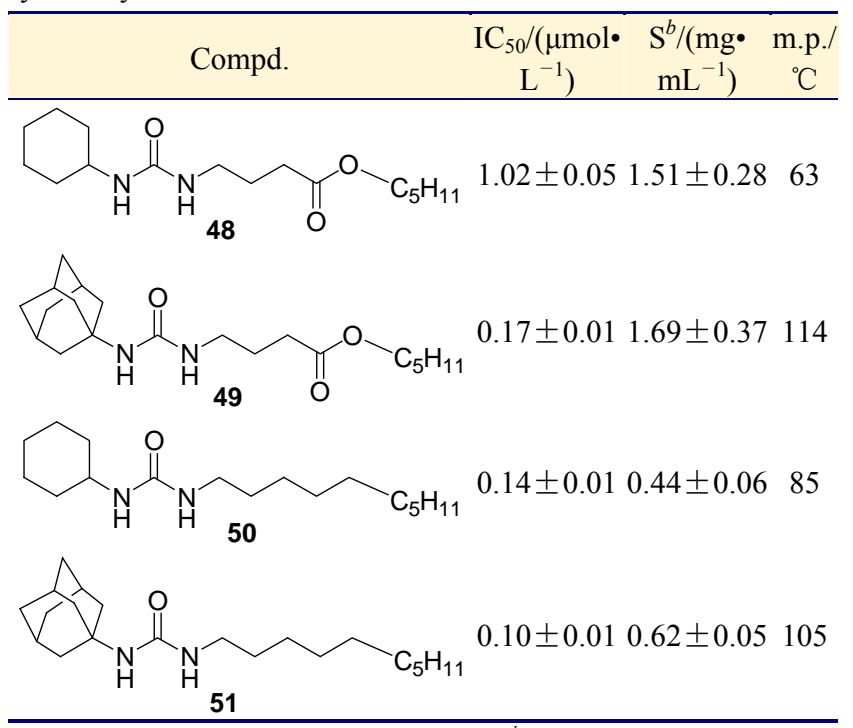

正因为分子中极性基团的增加可以改善化合物的 成药物理性质, 但活性有所下降, 所以 Kim 等继续寻找 可包含另一个极性基团的位置. 研究人员在先前的研究 中发现, 在距离脲羰基 5 个碳原子更远的区域(距离脲 羰基约 10１4 个碳原子)引入极性基团，活性保留且水 溶性提高, 这实际上类似于模拟环氧花生四烯酸的结 构, 引入长链的脂肪酸(化合物 $32 \sim 38$ ). 于是 $\mathrm{Kim}$ 等 $^{[16]}$ 在化合物 38 (AUDA)的基础上，一方面将末端羧基修饰 成相应的酯，增加化合物在油中的溶解度(通过皮下注 射或者局部给药, 改善的油溶性有利于药物的缓释制 剂); 另一方面将末端羧基修饰成相应的在体内更加稳 定的极性酰胺键, 增加化合物在水中的溶解度(Scheme $1)$.

在羧基的酯化修饰方面，长链取代要优于短链取 代，支链取代好于无支链的酯. 总体而言，这类酯化的 抑制剂活性保持, 水溶性较 AUDA 下降, 熔点下降, 油 溶性增大 (最高为 $27 \mathrm{mg} / \mathrm{mL}$, AUDA 为 $5 \mathrm{mg} / \mathrm{mL}$ ), 这为 给药方式多样性提供了有效的支撑. 在随后的药动学研 究中清楚地显示 AUDA 从这类化合物中释放出来, 即 这类 AUDA 的酯类化合物可以作为 AUDA 很好的软药 (soft drug). 这类化合物的药动学性质相较于 AUDA 略 微改善(最高血药浓度和曲线下面积), 但它们在体内的 半衰期却没有得到改善. 酰胺化修饰或末端引入糖, 都 没有很好改善这类化合物的理化性质.

Hammock 等 ${ }^{[28]}$ 还在化合物 49 的基础上, 保留距离 腿药效团 5 个碳原子处的极性基团的同时，探索引入的 第二个极性基团在抑制剂中的适当位置，以期在保持活 性的同时, 进一步改善此类抑制剂的成药物理性质(表 6). 结果发现，当第二个极性基团位于距脲羰基 13 16 个碳原子(化合物 56 59) 时, 活性不但没有下降, 熔点 还大幅降低. 根据计算出来的 $c \lg p$ 值和熔点，大致可以 推断出这些化合物的水溶性要好于化合物 49.

$\overline{{ }^{a} \text { 活性的测试采用了基于 } \mathrm{NEPC} \text { 的测试方法. }{ }^{b} 25{ }^{\circ} \mathrm{C} \text {, 在磷酸缓冲溶液 }(0.1}$ $\left.\mathrm{mol} \cdot \mathrm{L}^{-1}, \mathrm{pH}=7.4\right)$ 中的水溶液.

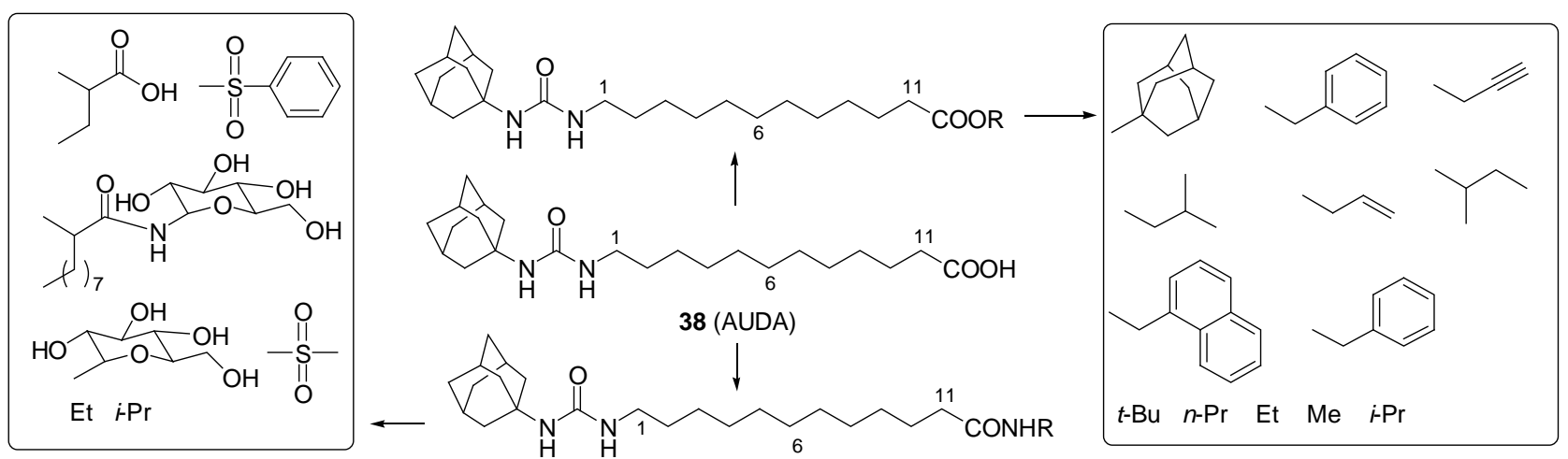

Scheme 1 
表 6 4-(3-金刚烷-1-基嫝基)丁酰基氧类化合物对 HsEH 的抑制活性 ${ }^{a}$

Table 6 Inhibition of HsEH by 4-(3-adamantan-1-ylureido)butyryloxy compounds<smiles>CCOC(=O)OCCCCNC(=O)NC12CC3CC(C1)C(C3)C2</smiles>

\begin{tabular}{cccccc}
\hline Compd. & $n$ & $\mathrm{IC}_{50} /\left(\mu \mathrm{mol} \cdot \mathrm{L}^{-1}\right)$ & $\mathrm{IC}_{90} /\left(\mu \mathrm{mol} \cdot \mathrm{L}^{-1}\right)$ & $\mathrm{m} . \mathrm{p} . /{ }^{\circ} \mathrm{C}$ & $c \lg p^{b}$ \\
\hline $\mathbf{5 2}$ & 1 & $0.39 \pm 0.01$ & $9 \pm 2$ & 123 & $0.98 \pm 0.47$ \\
$\mathbf{5 3}$ & 2 & $0.54 \pm 0.05$ & $9 \pm 21$ & $95 \sim 97$ & $1.27 \pm 0.47$ \\
$\mathbf{5 4}$ & 3 & $0.12 \pm 0.01$ & $5.0 \pm 0.1$ & $89 \sim 91$ & $1.55 \pm 0.47$ \\
$\mathbf{5 5}$ & 4 & $0.13 \pm 0.01$ & $1.5 \pm 0.1$ & $84 \sim 86$ & $1.97 \pm 0.47$ \\
$\mathbf{5 6}$ & 6 & $0.12 \pm 0.01$ & $0.81 \pm 0.01$ & $65 \sim 67$ & $2.81 \pm 0.47$ \\
$\mathbf{5 7}$ & 7 & $0.11 \pm 0.01$ & $0.72 \pm 0.01$ & $58 \sim 59$ & $3.22 \pm 0.47$ \\
$\mathbf{5 8}$ & 9 & $0.10 \pm 0.01$ & $0.68 \pm 0.01$ & $60 \sim 61$ & $4.06 \pm 0.47$ \\
$\mathbf{5 9}$ & 10 & $0.10 \pm 0.01$ & $1.2 \pm 0.1$ & $54 \sim 55$ & $4.48 \pm 0.47$ \\
$\mathbf{6 0}$ & 11 & $0.10 \pm 0.01$ & $1.8 \pm 0.2$ & $64 \sim 65$ & $4.89 \pm 0.47$ \\
\hline
\end{tabular}

${ }^{a}$ 活性的测试采用了基于 NEPC 的测定方法. ${ }^{b}$ 指计算获得的 $\lg p$ 值.

通过对这些化合物结构和活性关系的研究, Hommock 等 ${ }^{[28]}$ 创造性地提出了人类可溶性环氧化物水解酶 抑制剂的药效模型：包括主要药效团(图 2 中 A 所标示 的区域), 以脲的结构活性最好, 一端为大的疏水性的 金刚烷基; 二级药效团(图 2 中 B 所标示的区域), 包含 一个极性的羰基，距离腿羰基约 5 6 原子处，能够增强 化合物的水溶性; 三级药效团(图 2 中 C 所标示的区域), 它也包含一个极性的羰基, 距离嫝羰基约 12 16 原子 处. 二、三级药效团的引入对活性影响不大, 主要是为 了提高化合物的选择性和水溶性.

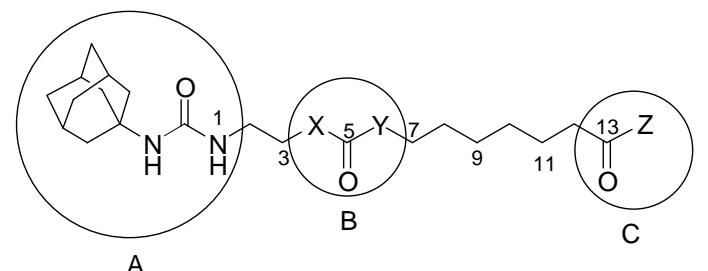

图 2 Hammock 工作组提出的可溶性环氧化物水解酶抑制剂 的药效团模型

Figure 2 sEH inhibitor pharmacophore model proposed by Hammock

在前期的实践中, 腿官能团改换成酰胺(表 1 , 化合 物 5)并未导致活性的急剧下降，并且相应的抑制剂的熔 点较脲类化合物要低, 而高熔点通常导致化合物在水和 有机溶剂中低的溶解度. 因此, 为了改善成药物理性质, Kim 等 ${ }^{[29]}$ 将腿改换成酰胺键, 同时保留金刚烷-1-基, 进 行了以酰胺为主要药效团的抑制剂的合成和活性评估 (表 7). 结果显示, 将腿官能团改换成酰胺键, 相应化合 物的活性普遍降低; 不过化合物 62 的活性下降幅度不 大, 但其熔点大大降低, 水溶性也大大增加. 其中, 艮
官能团中的 NH 保留在右边的活性比相应的保留在左边 的活性高(化合物 62 和 61). 虽然酯官能团在体内不稳 定，最终不能提供有效的血药浓度 ${ }^{[29]}$, 但是这类化合物 仍然可以通过皮下、气雾或者肠粘膜给药直接作用于靶 组织而作为软药使用.

从 $\mathrm{sEH}$ 的晶体结构来看, 虽然位于催化残基周边的 催化通道是限定在一定范围之内的, 但是当底物从反应 位点离去时却观察到催化通道的扩张. 这一结构特点表 明该酶可能允许主要烷基长链上存在合适的分支 ${ }^{[30]}$. 由于氨基酸是简单的具有多种不同边链的双官能团合 成子, Morisseau 等 ${ }^{[31]}$ 合成了含有单或二肽的脲类化合 物. 另外，在一些化合物中，氨基酸的存在通常能够大 大提高其生物利用度，这是因为肠中存在特异的二/三 肽转移物质. 因此, 通过对嫝药效团与氨基酸之间长度 以及氨基酸种类的笁选, Morisseau等获得了活性最好的 化合物 67(图 3), 其水溶性较化合物 37(AUDA)提高 10 倍以上, 然而在狗模型中，口服给药的药动学性质并不 理想.

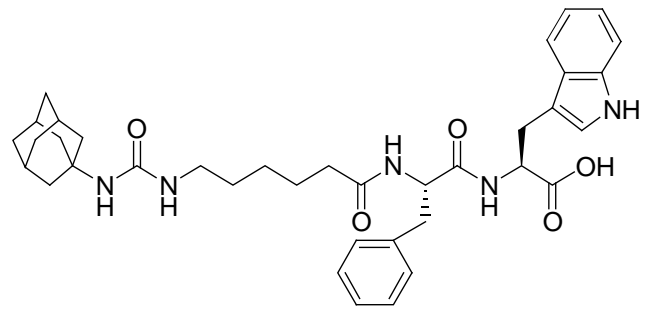

图 3 化合物 $\mathbf{6 7}$ 的结构

Figure 3 Structure of compound $\mathbf{6 7}$

\section{4 第三代 HsEH 抑制剂}

第二代 $\mathrm{HsEH}$ 抑制剂, 即刚性大基团加上(非)极性 柔性基团，具有较好的体内活性，有的水溶性也大幅度 
表 7 金刚烷基酰胺类化合物对 $\mathrm{HsEH}$ 的抑制活性 ${ }^{a}$

Table 7 Inhibition of HsEH by adamantan-1-yl acyl

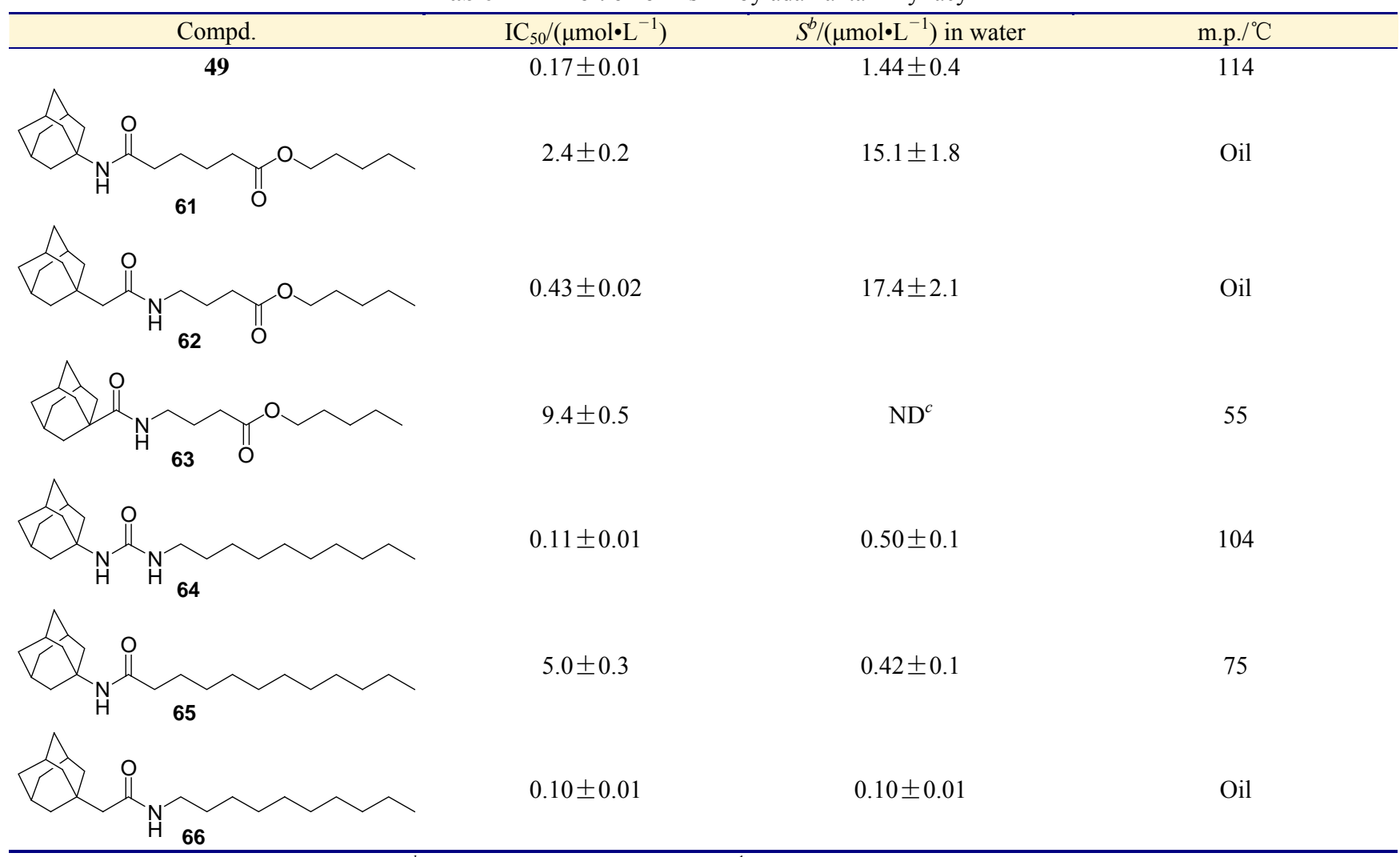

$\bar{a}$ 活性的测试采用了基于 $\mathrm{NEPC}$ 的测定方法; ${ }^{b} 25{ }^{\circ} \mathrm{C}$, 在磷酸缓冲溶液 $\left(0.1 \mathrm{~mol} \cdot \mathrm{L}^{-1}, \mathrm{pH}=7.4\right)$ 中的水溶性. ${ }^{c}$ 未测试.

提高, 但是柔性边链的存在导致这类化合物在体内迅速 地代谢. 因此, 引入一个构象限制(非柔性)的二级药效 团有可能改善其药动学性质, 同时又具有可观的体内活 性，这就是第三代 HsEH 抑制剂.

Jones 等 ${ }^{[32]}$ 为了扩展抑制剂的结构类型, 取得更详 尽的构效关系, 同时也为了增加类药性(drug-like), 选 用了构象限制的哌啶环作为二级药效团(表 8).

这一系列化合物的活性显示, 易于质子化的哌啶环 (由 $\mathrm{N}$ 原子质子化导致)与酶并不相容, 使得抑制剂的活 性低 $(68 \sim 71)$, 大多数都是微摩尔水平. 化合物 $72 \mathrm{a}$ 和 72b 却例外, 可能是因为作用位点上芳环-芳环的相互作 用抵消了阳离子的去稳定作用, 因此, 降低 $\mathrm{N}$ 原子质子 化的可能性也许就能提高活性. 最直接和方便的方法即 在此处引入酰胺键(表 9).

哌啶环的氮原子引入酰胺键确实大大提高了抑制 剂的活性, 其 $\mathrm{IC}_{50}$ 值均达到了纳摩尔水平. 这一系列化 合物 74 80 中 $n=0$ 的活性普遍高于 $n=1$. 当 $n=0$ 时, 酰胺键正好距艮羰基 5 个原子, 符合 Hammock 提出的 药效团模型. 化合物 76a 是这一系列抑制剂中活性最好 的化合物, 经过计算显示, 此处可能存在一个与三氟甲 基上的氟原子形成氢键的氨基酸残基 $\mathrm{Gln}^{382}$. 然而, 动
表 8 含有烷基哌啶结构的 1-金刚烷-1 基腿类化合物对 HsEH 的抑制活性 ${ }^{a}$

Table 8 Inhibition of $\mathrm{HsEH}$ by alkylpiperidine-containing 1-adamantan-1-yl ureas

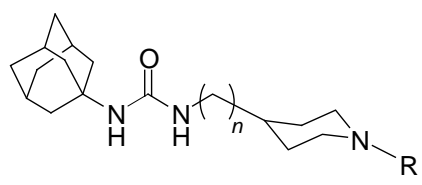

\begin{tabular}{|c|c|c|c|}
\hline Compd. & $\mathrm{R}$ & $n$ & $\mathrm{IC}_{50} /\left(\mu \mathrm{mol} \cdot \mathrm{L}^{-1}\right)$ \\
\hline $68 a$ & $\mathrm{H}$ & 0 & 0.3 \\
\hline $68 \mathrm{~b}$ & $\mathrm{H}$ & 1 & 4.2 \\
\hline $69 a$ & & 0 & 3.8 \\
\hline $69 b$ & & 1 & 3.9 \\
\hline $70 a$ & & 0 & 0.81 \\
\hline $70 \mathrm{~b}$ & & 1 & 2.6 \\
\hline $71 a$ & & 0 & 1.2 \\
\hline $71 b$ & & 1 & 0.61 \\
\hline $72 a$ & & 0 & 0.01 \\
\hline $72 b$ & & 1 & 0.11 \\
\hline
\end{tabular}

${ }^{a}$ 活性的测试采用 CMNPC 为底物, 基于荧光的测试方法. 
表 9 含有酰基哌啶结构的 1-金刚烷-1 基脲类化合物对 HsEH 的抑制活性 ${ }^{a, b}$

Table 9 Inhibition of HsEH by acylpiperidine-containing 1-adamantan-1-yl ureas

\begin{tabular}{|c|c|c|c|c|}
\hline Compd. & $n$ & $\mathrm{R}$ & $\mathrm{IC}_{50} /\left(\mathrm{nmol} \cdot \mathrm{L}^{-1}\right)$ & $\mathrm{AUC}^{c} /\left(10^{4} \mathrm{nmol} \cdot \mathrm{L}^{-1} \cdot \min \right)$ \\
\hline $73 a$ & 0 & $\mathrm{CH}_{3}$ & 7.0 & 3.7 \\
\hline $73 b$ & 1 & $\mathrm{CH}_{3}$ & 5.0 & 0.55 \\
\hline $74 a$ & 0 & $\mathrm{CH}_{2} \mathrm{CH}_{3}$ & 3.2 & 0.65 \\
\hline $74 b$ & 1 & $\mathrm{CH}_{2} \mathrm{CH}_{3}$ & 8.7 & \\
\hline $75 a$ & 0 & $\mathrm{CH}_{2} \mathrm{CH}_{2} \mathrm{CH}_{3}$ & 2.6 & 0.25 \\
\hline $75 b$ & 1 & $\mathrm{CH}_{2} \mathrm{CH}_{2} \mathrm{CH}_{3}$ & 6.7 & \\
\hline 76a & 0 & $\mathrm{CF}_{3}$ & 1.1 & 0.33 \\
\hline $76 b$ & 1 & $\mathrm{CF}_{3}$ & 1.8 & \\
\hline $77 a$ & 0 & $\mathrm{Ph}$ & 1.3 & 0.061 \\
\hline $77 b$ & 1 & $\mathrm{Ph}$ & 3.2 & 0.033 \\
\hline $78 a$ & 0 & & 1.2 & 0.47 \\
\hline $78 b$ & 1 & & 7.6 & \\
\hline 79a & 0 & & 1.7 & \\
\hline 79b & 1 & & 5.4 & \\
\hline 80a & 0 & & 2.1 & \\
\hline 80b & 1 & & 7.3 & \\
\hline $37(\mathrm{AU}$ & & & & 0.31 \\
\hline
\end{tabular}

${ }^{a}$ 活性的测试采用 $\mathrm{CMNPC}$ 为底物, 基于苂光的测试方法. ${ }^{b}$ 通过口服给药狗模型获得 $(0.3 \mathrm{mg} / \mathrm{kg})$, 药-时曲线下面积.

物体内药代动力学试验显示, 活性最好的化合物 76a 的 血药浓度相对化合物 37 (AUDA)并未得到改善，而活性 相对较差的化合物 73a 的血药浓度却是 AUDA 的 12 倍 (表 9). 总体而言, 该类抑制剂在体内的半衰期同样很 短, 并且血药浓度也比较低.

在许多生物活性分子中都包含有哌嗪这一杂环结 构. 哌嗪具有很好的成药物理性质, 尤其显示在水溶性 方面. 另一方面, 哌嗪环作为构象限制的结构引入到活 性分子中, 有可能增强化合物的药代稳定性. 我们课题 组 ${ }^{[33]}$ 以天然氨基酸为起始原料, 合成了四个将哌嗪环 置入二级官能团的 HsEH 抑制剂(表 10). 虽然这一系列 化合物的抑制活性与对照化合物相比下降了许多, 但是 水溶性大幅提高、熔点大幅降低, 可见在改善成药物理 性质方面，哌嗪环确实起到很大的作用.

$\mathrm{HsEH}$ 抑制剂是一个亲脂性抑制剂, 极性的增加有
利于物理性质的改善, 但却降低了活性. 为了平衡二者 的关系, 我们课题组 ${ }^{[34]}$ 又将哌嗪环置入第三药效团并 对第二药效团组合进行扫描，果然获得了水溶性大幅提 高的一系列 $\mathrm{IC}_{50}$ 值小于 $10 \mathrm{nmol} \cdot \mathrm{L}^{-1}$ 的 $\mathrm{HsEH}$ 抑制剂. 初 步的大鼠模型口服给药实验显示这些活性化合物具有 极好的药动学性质, 如 N(4)为乙酰基取代的化合物 90 的 $\mathrm{IC}_{50}$ 为 $7 \mathrm{nmol} \cdot \mathrm{L}^{-1}$, 半衰期为 $14 \mathrm{~h}$, 药-时曲线下面积 (AUC)达 $40200 \mathrm{nmol} \cdot \mathrm{L}^{-1} \cdot \min ($ 表 11), 具有进一步开发 的潜力.

\section{$1.5 \mathrm{HsEH}$ 抑制剂近期研究进展}

近两三年来，一些课题组继续对各个药效团之间的 连接体做了进一步的研究和拓展, 例如用水杨酸连接主 要药效团和二级药效团，进一步丰富了这类抑制剂的结 构类型 ${ }^{[35]}$. 还有一些研究旨在寻找金刚烷基的替代基 团，例如 Anandan 等 ${ }^{[36]}$ 用一系列的取代芳基置换金刚烷 
表 10 含哌嗪结构的 1-金刚烷基脲类化合物的抑制活性及其相关的物理性质 ${ }^{a}$

Table 10 Inhibition of HsEH by piperazine-containing 1-adamantyl ureas and the related physical properties

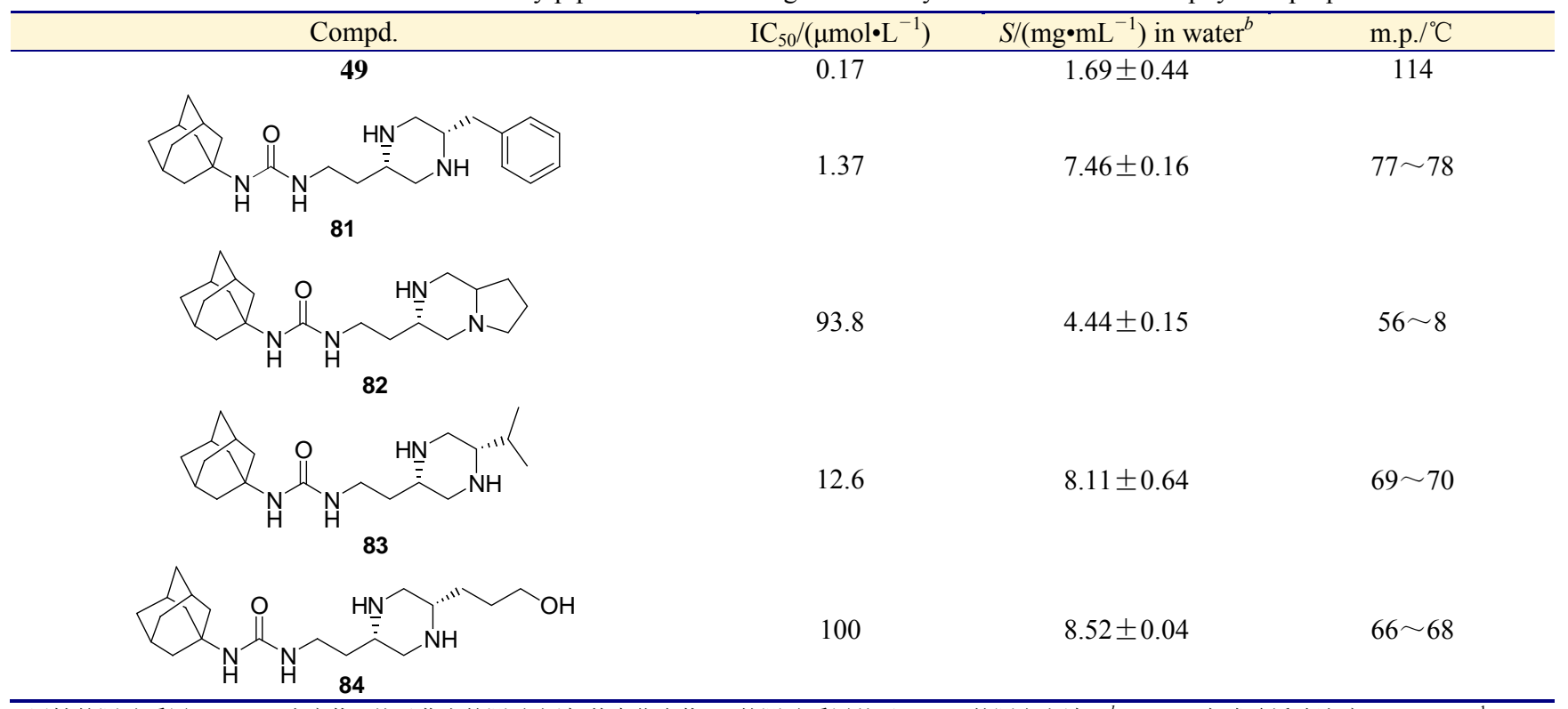

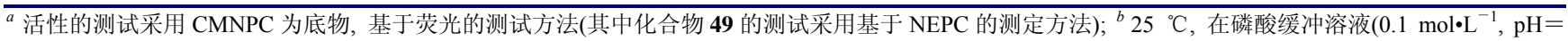
7.4)中的水溶性.

表 11 苯环上不同位置以及哌嗪-4-基上不同取代基的 1-金刚烷-1-基-3-(哌嗪-1-基烷氧苯基)腿衍生物对 HsEH 的抑制活性及其在 小鼠体内的药动学参数 ${ }^{a}$

Table 11 Inhibition of HsEH and in vivo pharmacokinetic parameters in mice of 1-adamantan-1-yl-3-(piperazin-1-ylalkoxyphenyl)urea derivatives with variation on the phenyl substitution position and piperazin-4-yl substitution

\begin{tabular}{cccccccc} 
& & & & & \\
& & & & \\
\hline
\end{tabular}

$\bar{a}$ 活性的测试采用 $\mathrm{CMNPC}$ 为底物, 基于苂光的测试方法(其中化合物 $\mathbf{4 9}$ 的测试采用基于 $\mathrm{NEPC}$ 的测定方法); ${ }^{b} 25{ }^{\circ} \mathrm{C}$, 在磷酸缓冲溶液 $\left(0.1 \mathrm{~mol} \cdot \mathrm{L}^{-1}, \mathrm{pH}=\right.$ 7.4)中的水溶性.

基, 发现芳基对位若以强吸电子基团 $\left(4-\mathrm{CF}_{3}, 4-\mathrm{CF}_{3} \mathrm{O}\right.$ 或 4-Cl)取代就能获得与金刚烷基取代的化合物相当的抑 制活性. Hammok 等 ${ }^{[37]}$ 在前期工作 ${ }^{[32]}$ 的基础上, 在保留 脲一侧 $\mathrm{N}$ 上取代基为 4-哌啶基的基础上，也对金刚烷基 的替代基团进行了详尽的研究, 结果也发现了一系列的 取代芳基 $\left(2,4-\mathrm{Cl}_{2}, 4-\mathrm{CF}_{3}, 4-\mathrm{CF}\left(\mathrm{CF}_{3}\right)_{2}\right.$ 等)能够很好地替代 金刚烷基发挥抑制活性.

Eldrup 等 ${ }^{[38}$ 通过高通量篎选, 还获得了含有哌啶基 脲为主要药效团的 $\mathrm{sEH}$ 抑制剂 91, 随后对哌啶上取代 基和嫝基团右边的部分进行修饰, 获得了活性和药动学 性质都非常好的化合物 92(图 4).

另外, 许多以非腿为主要官能团的 sEH 抑制剂也被 发现和发展起来. 例如 Nishi 等 ${ }^{[39]}$ 在 Hammock 等 ${ }^{[29]}$ 工作

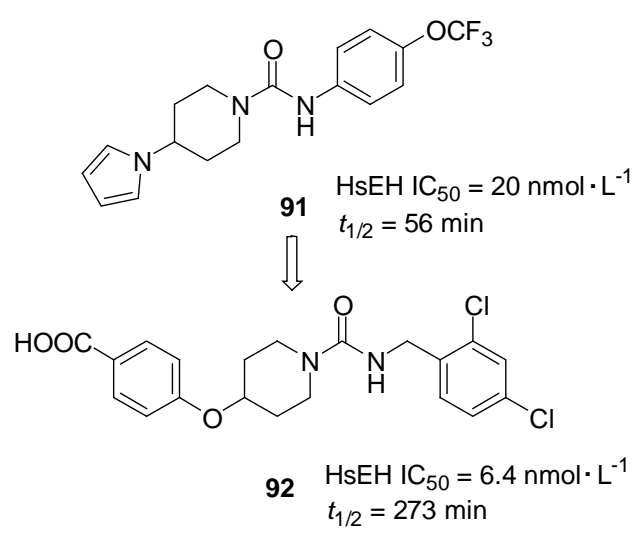

图 4 化合物 91 和 92 的结构

Figure 4 Structures of compounds $\mathbf{9 1}$ and $\mathbf{9 2}$ 
的基础上, 进一步研究了以酰胺为主要官能团的 HsEH 抑制剂, 发现以环烷基取代的酰胺为主要药效团的化合 物不仅具有与以脲为主要官能团抑制剂相当的活性, 成 药性质也大大改善. Landry 等 ${ }^{[00]}$ 通过高通量笁选获得了 一个酰胺类的 sEH 抑制剂先导化合物 93, 随后还对酰胺 $\mathrm{N}$ 的取代基和哌啶 $\mathrm{N}$ 的取代基进行了一系列的改造和修 饰, 这一系列活性最好的化合物 94 同样也是以环烷基取 代的酰胺化合物, 其 $\mathrm{IC}_{50}$ 值为 $7.9 \mathrm{nmol} \cdot \mathrm{L}^{-1}$, 不过作者并 未给出任何的水溶性、熔点或是药动学性质的数据(图 5).<smiles>Cc1cc(C)c(S(=O)(=O)N2CCC(C(=O)NCc3ccc(Cl)cc3Cl)CC2)c(C)c1</smiles>
$\mathrm{IC}_{50}=20 \mathrm{nmol} \cdot \mathrm{L}^{-1}$
Taylor 等 ${ }^{[41]}$ 通过高通量篮选获得三个不同类型的 先导化合物，他综合考虑其中两种酰胺类 sEH 抑制剂 (95 和 96)在活性和药动学性质上的各自的优势, 将其中 一类的酰基部分与另一类的氨基部分拼合起来，经过一 系列的优化，最终获得了活性和药动学性质有所改善的 化合物(图 6).

Eldrup 等 ${ }^{[42]}$ 则在 96 的基础上，优化酰胺两侧的取 代基，获得了高抑制活性、高稳定性以及良好药动学性 质的抑制剂 97(图 7).

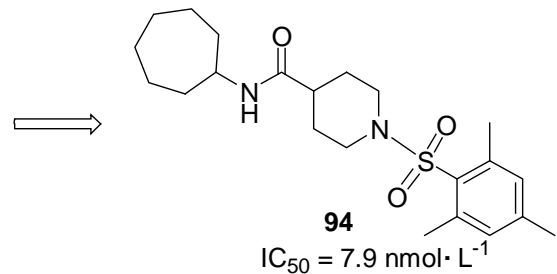

图 5 化合物 93 和 94 的结构

Figure 5 Structures of compounds 93 and 94<smiles>O=C(NC1CCOCC1)C1CCN(C(=O)NCc2ccc(Cl)cc2Cl)CC1</smiles>

$\mathrm{HsEH} \mathrm{IC}{ }_{50}=180 \mathrm{nmol} \cdot \mathrm{L}^{-1}$ $t_{1 / 2}=300 \mathrm{~min}$<smiles>O=C(NCCC(c1ccccc1)c1ccccc1)c1cccnc1</smiles><smiles>[R]c1ccc(C(=O)NCc2ccc(Cl)cc2Cl)cn1</smiles><smiles>[R]c1ccc(CNC(=O)c2ccc(OC(F)(F)F)nc2)c([R])c1</smiles>

$\mathrm{HsEH} \mathrm{IC}{ }_{50}=7 \mathrm{nmol} \cdot \mathrm{L}^{-1}$

$t_{1 / 2}=7 \mathrm{~min}$

图 6 将嫝系列(95)的右半部分与烟酰胺(96)的左半部分拼合起来获得的抑制剂保留了各系列的优势

Figure 6 A combination of the right-hand side of the urea series (95) coupled with the left-hand side of the nicotinamides (96) provides inhibitors that retain the optimal properties from each of the parent series<smiles>O=C(NCCC(c1ccccc1)c1ccccc1)c1cccnc1</smiles>

$\mathrm{HsEH} \mathrm{IC}_{50}=7 \mathrm{nmol} \cdot \mathrm{L}^{-1}$ $t_{1 / 2}=7 \mathrm{~min}$ cell-based $\mathrm{IC}_{50}=9 \mathrm{nmol} \cdot \mathrm{L}^{-1}$<smiles>C=CCCCC(c1ccc(F)cc1)c1ccc(S(C)(=O)=O)cc1</smiles>

$\mathrm{HsEH} I \mathrm{C}_{50}=6 \mathrm{nmol} \cdot \mathrm{L}^{-1}$

$t_{1 / 2}>300 \mathrm{~min}$

cell-based $\mathrm{IC}_{50}<0.14 \mathrm{nmol} \cdot \mathrm{L}^{-1}$

图 7 化合物 96 和 97 的结构

Figure 7 Structures of compounds $\mathbf{9 6}$ and $\mathbf{9 7}$ 
值得一提的是，各大制药公司近几年也都针对 $\mathrm{sEH}$ 抑制剂展开了研究. 例如 Merck 公司先后开发了四类 sEH 抑制剂 ${ }^{[43]}$ (图 8), 其中三个系列中最优的化合物不 仅具有优异的酶抑制活性和体内药动学性质, 并且能够 很好地区分其它类别的 CYP(细胞色素 P450), 同时对 $\mathrm{Na}, \mathrm{K}$ 及钙离子通道的影响也较小. 其它如辉瑞 ${ }^{[4]}$ 和日 本住友制药 ${ }^{[4]}$ 也在这一领域开展了研究.
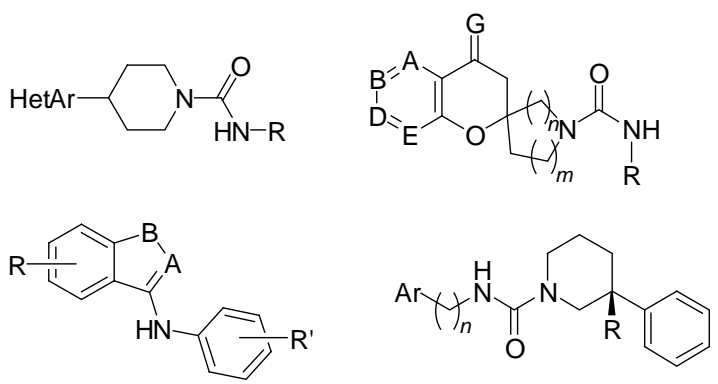

图 8 默克公司开发的四类 $\mathrm{sEH}$ 抑制剂的骨架

Figure 8 The four scaffolds of sEH inhibitor developed by Merck

\section{2 结论和展望}

由于可溶性环氧化物水解酶的广泛存在，可溶性环 氧化物水解酶抑制剂可用于治疗由可溶性环氧化物水 解酶失调引起的疾病, 包括治疗或者减缓肾恶化、高血 压、血管炎症、阻塞性肺病、间质性肺病以及哮喘等. 不 过目前的研究重点主要集中于高血压以及由此引发的 心血管疾病的治疗.

从早期的环氧类人类可溶性环氧化物水解酶抑制 剂的发现, 到第三代脲类人类可溶性环氧化物水解酶抑 制剂的设计合成, 人类可溶性环氧化物水解酶抑制剂的 研究经历了 30 多年的发展. 从最初的只具备体外微摩 尔级抑制活性、体内极差甚至无活性的 HsEH 抑制剂发 展到目前的体外纳摩尔级活性、体内具备优异的药动学 性质的 HsEH 抑制剂. 对人类可溶性环氧化物水解酶的 了解促进了其抑制剂设计的合理性, 尤其是 Hammoc 等 创造性地提出的人类可溶性环氧化物水解酶抑制剂的 药效模型，使得 HsEH 抑制剂的设计合成更加合理可行.

随着药理学的发展, 人类可溶性环氧化物水解酶的 作用和机理正在不断地被发现和阐明, 也进一步证明了 其抑制剂作为治疗高血压的合理性和有效性. 随着研究 的推进，将会有越来越多的、也更加优异的人类可溶性 环氧化物水解酶抑制剂被设计和合成出来, 这将可能会 为高血压以及炎症相关疾病的治疗提供另一条可行的 途径.

\section{References}

[1] Jerina, D. M. Lloydia 1974, 37, 212.

[2] Oliw, E. H. Prog. Lipid Res. 1994, 33, 329.

[3] Morisseau, C. Hammock, B. D. Annu. Rev. Pharmacol. Toxicol. 2005, 45, 311.

[4] Hammock, B. D.; Grant, D.; Storms, D. In Comprehensive Toxicology, Eds.: Sipes, I.; McQueen, C.; Gandolfi, A., Pergamon, New York, 1997, 283.

[5] Oesch, F. Xenobiotica 1973, 3, 305.

[6] Levin, W.; Michaud, D. P.; Thomas, P. E.; Jerina, D. M. Arch. Biochem. Biophys. 1983, 220, 485.

[7] Pace-Asciak, C. R.; Lee, W. S. J. Biol. Chem. 1989, 264, 9310.

[8] (a) Yu, Z.; Xu, F.; Huse, L. M.; Morisseau, C.; Draper, A. J. Circ. Res. 2000, 87, 992.

(b) Davis, B. B.; Thompson, D. A.; Howard, L. L.; Morisseau, C.; Hammock, B. D. Proc. Natl. Acad. Sci U. S. A. 2002, 99, 2222.

(c) Imig, J. D.; Zhao, X.; Capdevila, J. H.; Morisseau, C.; Hammock, B. D. Hypertension 2002, 39, 690.

(d) Zhao, X.; Yamamoto, T.; Newman, J. W.; Kim, I. H.; Watanabe, T. J. Am. Soc. Nephrol. 2004, 15, 1244.

[9] Arand, M.; Grant, D. F.; Beetham, J. K.; Friedberg, T.; Oesch, F.; Hammock, B. D. FEBS Lett. 1994, 338, 251.

[10] Ollis, D. L.; Cheah, E.; Cygler, M.; Dijkstra, B.; Frolow, F. Curr. Protein Pept. Sci. 2000, 1, 209.

[11] Moghaddam, M. F.; Grant, D. F.; Cheek, J. M.; Greene, J. F.; Williamson, K. C.; Hammock, B. D. Nat. Med. 1997, 3, 562.

[12] Morisseau, C.; Goodrow, M. H.; Dowdy, D.; Zheng, J.; Greene, J. F.; Sanborn, J. R.; Hammock, B. D. Proc. Natl. Acad. Sci. U. S. A. 1999, 96, 8849.

[13] Spector, A. A.; Fang, X.; Snyder, G. D.; Weintraub, N. L. Prog. Lipid Res. 2004, 43, 55.

[14] (a) Imig, J. D.; Zhao, X.; Zaharis, C. Z.; Olearczyk, J. J.; Pollock, D. M.; Newman, J. W.; Kim, I.-H.; Watanabe, T.; Hammock, B. D. Hypertension 2005, 46, 975.

(b) Ai, D.; Fu, Y.; Guo, D.; Tanaka, H.; Wang, N.; Tang, C.; Hammock, B. D.; Shyy, J. Y.-J.; Zhu, Y. Proc. Natl. Acad. Sci. U. S. A. 2007, 104, 9018

[15] Sinal, C. J.; Miyata, M.; Tohkin, M.; Nagata, K.; Bend, J. R.; Gonzalez, F. J. J. Biol. Chem. 2000, 275, 40504.

[16] Kim, I.-H.; Nishi, K.; Tsai, H.-J.; Bradford, T.; Koda, Y.; Watanabe, T.; Morisseau, C.; Blanchfield, J.; Tothb, I.; Hammock, B. D. Bioorg. Med. Chem. 2007, 15, 312.

[17] The ALLHAT Officers and Coordinators for the ALLHAT Collaborative Research Group J. Am. Med. Assoc. 2002, 288, 2981.

[18] Schmelzer, K. R.; Inceoglu, B.; Kubala, L.; Kim, I. H.; Jinks, S. L.; Eiserich, J. P.; Hammock, B. D. Proc. Natl. Acad. Sci. U. S. A. 2006, 103, 13646.

[19] Mullin, C. A.; Hammock, B. D. 1982, 216, 423.

[20] Dietze, E. C.; Kuwano, E.; Casas, J.; Hammock, B. D. Biochem. Pharmacol. 1991, 42, 1163.

[21] Morisseau, C.; Du, G.; Newman, J. W.; Hammock, B. D. Arch. Biochem. Biophys. 1998, 356, 214.

[22] Draper, A. J.; Hammock, B. D. Toxicol. Sci. 1999, 52, 26.

[23] Gaetke, L. M.; McClain, C. J.; Talwalkar, R. T.; Shedlofsky, S. I. Am. J. Physiol. Endocrin. Metab. 1997, 272, E952.

[24] Dietze, E. C.; Kuwano, E.; Hammock, B. D. Anal. Biochem. 1994, 216, 176.

[25] Jones, P. D.; Wolf, N. M.; Morisseau, C.; Whetstone, P.; Hock, B.; Hammock, B. D. Anal. Biochem. 2005, 343, 66.

[26] Argiriadi, M. A.; Morisseau, C.; Hammock, B. D.; Christianson, D. W. Proc. Natl. Acad. Sci. U. S. A. 1999, 96, 10637. 
[27] Morisseaua, C.; Goodrow, M. H.; Newman, J. W.; Wheelock, C. E.; Dowdy, D. L.; Hammock, B. D. Biochem. Pharmacol. 2002, 63, 1599.

[28] Kim, I.-H.; Morisseau, C.; Watanabe, T.; Hammock, B. D. J. Med. Chem. 2004, 47, 2110.

[29] Kim, I.-H.; Heirtzler, F. R.; Morisseau, C.; Nishi, K.; Tsai, H.-J.; Hammock, B. D. J. Med. Chem. 2005, 48, 3621.

[30] (a) Argiriadi, M. A.; Morisseau, C.; Goodrow, M. H.; Dowdy, D. L.; Hammock, B. D.; Christianson, D. W. J. Biol. Chem. 2000, 275, 15265.

(b) Gomez, G. A.; Morisseau, C.; Hammock, B. D.; Christianson, D. W. Biochemistry 2004, 43, 4716.

[31] Morisseau, C.; Newman, J. W.; Tsai, H.-J.; Baecker P. A.; Hammock, B. D. Bioorg. Med. Chem. Lett. 2006, 16, 5439.

[32] Jones, P. D.; Tsai, H.-J.; Do, Z. N.; Morisseau, C.; Hammock, B. D. Bioorg. Med. Chem. Lett. 2006, 16, 5212.

[33] Li, H.-Y.; Jin, Y.; Morisseau, C.; Bruce, D.; Hammock, B. D.; Long, Y.-Q. Bioorg. Med. Chem. 2006, 14, 6586.

[34] Huang, S.-X.; Li, H.-Y.; Liu, J.-Y.; Morisseau, C.; Hammock, B. D.; Long, Y.-Q. J. Med. Chem. 2010, 53, 8376.

[35] Kasagami, T.; Kim, I.-H.; Tsai, H.-J.; Nishi, K.; Hammock, B. D.; Morisseau, C. Bioorg. Med. Chem. Lett. 2009, 19, 1784.

[36] Anandan, S.-K.; Webb, H. K.; Do, Z. N.; Gless, R. D. Bioorg. Med. Chem. Lett. 2009, 19, 4259.

[37] Rose, T. E.; Morisseau, C.; Liu, J.-Y.; Inceoglu, B.; Jones, P. D.; Sanborn, J. R.; Hammock, B. D. J. Med. Chem. 2010, 53, 7067.

[38] Eldrup, A. B.; Soleymanzadeh, F.; Farrow, N. A.; Kukulka, A.; De Lombaert, S. Bioorg. Med. Chem. Lett. 2010, 20, 571.

[39] Kim, I.-H.; Park, Y.-K.; Hammock, B. D.; Nishi, K. J. Med. Chem. 2011, 54, 1752.

[40] Xie, Y.; Liu, Y.; Gong, G.; Smith, D. H.; Yan, F.; Rinderspacher, A.; Feng, Y.; Zhu, Z.; Li X.; Deng, S.-X.; Branden, L.; Vidović, D.;
Chung, C.; Schürer, S.; Morisseau, C.; Hammock, B. D.; Landry, D. W. Bioorg. Med. Chem. Lett. 2009, 19, 2354.

[41] Taylor, S. J.; Soleymanzadeh, F.; Eldrup, A. B.; Farrow, N. A.; Muegge, I.; Kukulka, A.; Kabcenell, A. K.; De Lombaert, S. Bioorg. Med. Chem. Lett. 2009, 19, 5864.

[42] Eldrup, A. B.; Soleymanzadeh, F.; Taylor, S. J.; Muegge, I.; Farrow, N. A.; Joseph, D.; McKellop, K.; Man, C. C.; Kukulka, A.; De Lombaert, S. J. Med. Chem. 2009, 52, 5880.

[43] (a) Shen, H. C.; Ding, F.-X.; Wang, S.; Deng, Q.; Zhang, X.; Chen, Y.; Zhou, G.; Xu, S.; Chen, H.-S.; Tong, X.; Tong, V.; Mitra, K.; Kumar, S.; Tsai, C.; Stevenson, A. S.; Pai, L.-Y.; Alonso-Galicia, M.; Chen, X.; Soisson, S. M.; Roy, S.; Zhang, B.; Tata, J. R.; Berger, J. P.; Colletti, S. L. J. Med. Chem. 2009, 52, 5009.

(b) Shen, H. C.; Ding, F.-X.; Wang, S.; Xu, S.; Chen, H.-S.; Tong, X.; Tong, V.; Mitra, K.; Kumar, S.; Zhang, X.; Chen, Y.; Zhou, G.; Pai, L.-Y.; Alonso-Galicia, M.; Chen, X.; Zhang, B.; Tata, J. R.; Berger, J. P.; Colletti, S. L. Bioorg. Med. Chem. Lett. 2009, 19, 3398.

(c) Shen, H. C.; Ding, F.-X.; Deng, Q.; Xu, S.; Tong, X.; Zhang, X.; Chen, Y.; Zhou, G.; Pai, L.-Y.; Alonso-Galicia, M.; Roy, S.; Zhang, B.; Tata, J. R.; Berger, J. P.; Colletti, S. L. Bioorg. Med. Chem. Lett. 2009, 19, 5716.

(d) Shen, H. C.; Ding, F.-X.; Deng, Q.; Xu, S.; Chen, H.-S.; Tong, X.; Tong, V.; Zhang, X.; Chen, Y.; Zhou, G.; Pai, L.-Y.; Alonso-Galicia, M.; Zhang, B.; Roy, S.; Tata, J. R.; Berger, J. P.; Colletti, S. L. Bioorg. Med. Chem. Lett. 2009, 19, 5314.

[44] Xing, L.; McDonald, J. J.; Kolodziej, S. A.; Kurumbail, R. G.; Williams, J. M.; Warren, C. J.; O'Neal, J. M.; Skepner, J. E.; Roberds, S. L. J. Med. Chem. 2011, 54, 1211.

[45] Tanaka, D.; Tsuda, Y.; Shiyama, T.; Nishimura, T.; Chiyo, N.; Tominaga, Y.; Sawada, N.; Mimoto, T.; Kusunose, N. J. Med. Chem. 2011, 54, 851 . 